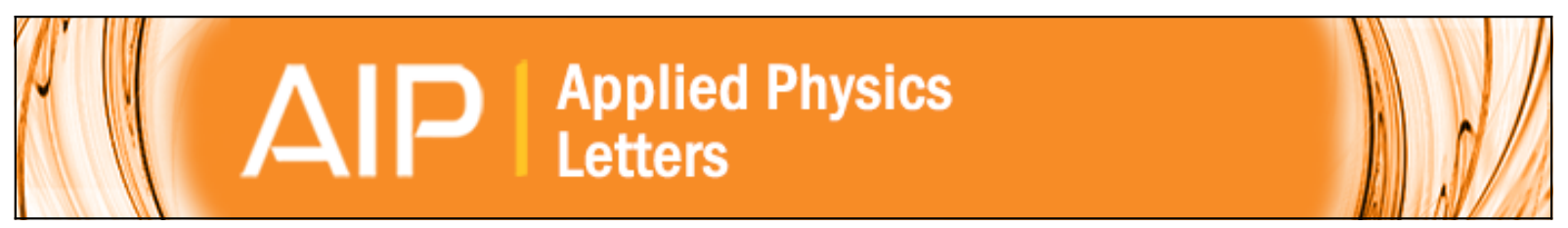

\title{
Cylindrical vector beams of light from an electrically excited plasmonic lens
}

Shuiyan Cao, Eric Le Moal, Elizabeth Boer-Duchemin, Gérald Dujardin, Aurélien Drezet, and Serge Huant

Citation: Applied Physics Letters 105, 111103 (2014); doi: 10.1063/1.4895769

View online: http://dx.doi.org/10.1063/1.4895769

View Table of Contents: http://scitation.aip.org/content/aip/journal/apl/105/11 ?ver=pdfcov

Published by the AIP Publishing

\section{Articles you may be interested in}

Dynamic plasmonic beam shaping by vector beams with arbitrary locally linear polarization states

Appl. Phys. Lett. 105, 011110 (2014); 10.1063/1.4887824

Interference of surface plasmon polaritons excited at hole pairs in thin gold films

Appl. Phys. Lett. 101, 201102 (2012); 10.1063/1.4767523

Colored porous silicon as support for plasmonic nanoparticles

J. Appl. Phys. 111, 084302 (2012); 10.1063/1.3703469

Spectral analysis of enhanced third harmonic generation from plasmonic excitations

Appl. Phys. Lett. 98, 261909 (2011); 10.1063/1.3604794

Subwavelength proximity nanolithography using a plasmonic lens

J. Vac. Sci. Technol. B 25, 2271 (2007); 10.1116/1.2804517

AIP $\left.\right|_{\text {Letters }} ^{\text {Applied Physics }}$

is pleased to announce Reuben Collins as its new Editor-in-Chief

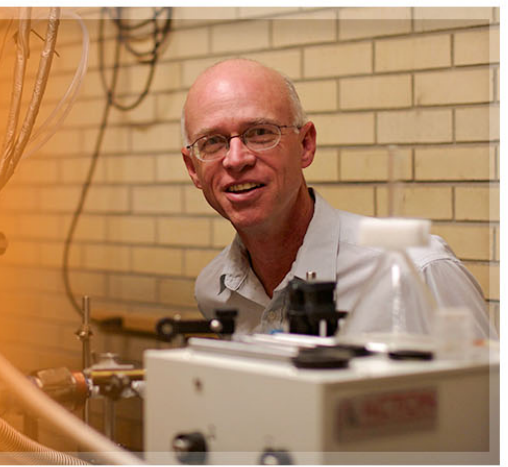




\title{
Cylindrical vector beams of light from an electrically excited plasmonic lens
}

\author{
Shuiyan Cao, ${ }^{1, a)}$ Eric Le Moal, ${ }^{1}$ Elizabeth Boer-Duchemin, ${ }^{1}$ Gérald Dujardin, ${ }^{1}$ \\ Aurélien Drezet, ${ }^{2,3}$ and Serge Huant ${ }^{2,3}$ \\ ${ }_{1}^{1}$ Institut des Sciences Moléculaires d'Orsay, CNRS - Université Paris-Sud (UMR 8214), Orsay, France \\ ${ }^{2}$ Université Grenoble Alpes, Inst. NEEL, F-38000 Grenoble, France \\ ${ }^{3}$ CNRS, Inst. NEEL, F-38042 Grenoble, France
}

(Received 25 August 2014; accepted 2 September 2014; published online 15 September 2014)

\begin{abstract}
The production of cylindrical vector beams from a low-energy, electric, microscale light source is demonstrated both experimentally and theoretically. This is achieved by combining a "plasmonic lens" with the ability to locally and electrically excite propagating surface plasmons on gold films. The plasmonic lens consists of concentric circular subwavelength slits that are etched in a thick gold film. The local excitation arises from the inelastic tunneling of electrons from the tip of a scanning tunneling microscope. We report on the emission of radially polarized beams with an angular divergence of less than $\pm 4^{\circ}$. ( $) 2014$ AIP Publishing LLC. [http://dx.doi.org/10.1063/1.4895769]
\end{abstract}

Cylindrical vector $(\mathrm{CV})$ beams are light beams that have spatially varying polarization properties of cylindrical symmetry and zero intensity along the propagation axis. ${ }^{1}$ The unique propagation, diffraction, and focusing properties of $\mathrm{CV}$ beams are attracting increasing interest, in particular for their use in the control of light-matter interactions on a submicrometer scale. ${ }^{2,3}$ Applications have been reported in various fields including quantum optics, ${ }^{4}$ high-resolution imaging, ${ }^{5}$ and in the trapping and manipulation of nanoobjects. ${ }^{6}$ Numerous optical methods have been reported either for the generation of $\mathrm{CV}$ beams using a macroscopic light source ${ }^{1}$ or for the conversion of light beams with homogeneous polarization into $\mathrm{CV}$ beams using birefringent materials, phase plates, spatial light modulators, ${ }^{7}$ subwavelength gratings, ${ }^{8}$ or metallic apertures. ${ }^{9}$ Comparatively, much less has been reported for electrically driven microscale light sources generating CV beams, ${ }^{10-12}$ though such sources are highly desirable for integrated applications, e.g., in data communications or optical sensors. An electrically driven metallic cavity nanolaser generating an azimuthally polarized light beam has been demonstrated recently, ${ }^{10}$ as well as microscale vertical cavity surface emitting lasers (VCSEL) incorporating surface gratings so as to yield either azimuthally ${ }^{11}$ or radially polarized beams. ${ }^{12}$ The applications of these sources are limited, however, by their relatively high beam divergence $\left( \pm 11.5^{\circ}\right.$ (Ref. 11) and $\pm 37^{\circ}$ (Ref. 10)).

In this Letter, we report on an original method for the electrical generation of radially polarized beams of very low divergence angle $\left( \pm 4^{\circ}\right)$ from a microscale plasmonic structure. As shown in Fig. 1(a), the plasmonic structure is a plasmonic lens ${ }^{13}$ consisting of concentric circular slits etched in a thick gold film. Local, low-energy, electrical excitation of surface plasmons (SPs) is achieved with tunnel electrons from the tip of a scanning tunneling microscope (STM) on the central disk region of the structure. The SPs propagate from the excitation site to the slits which then scatter the SPs into light. The resulting emitted radiation is radially polarized and forms a cylindrical beam with a diameter that is

a) shuiyan.cao@u-psud.fr approximately that of the central disk of the plasmonic lens. In this work, we experimentally and theoretically study how the central disk diameter, the number of slits and the excitation site affect the divergence angle. We show that by changing the excitation position of the tip we can vary the emission direction up to about $10^{\circ}$ from the normal while preserving a beam of low divergence angle $\left( \pm 6^{\circ}\right)$. However, the polarization state is gradually modified as the tip is moved off-center, thus degrading the pure $\mathrm{CV}$ nature of the light, while maintaining a highly collimated beam.
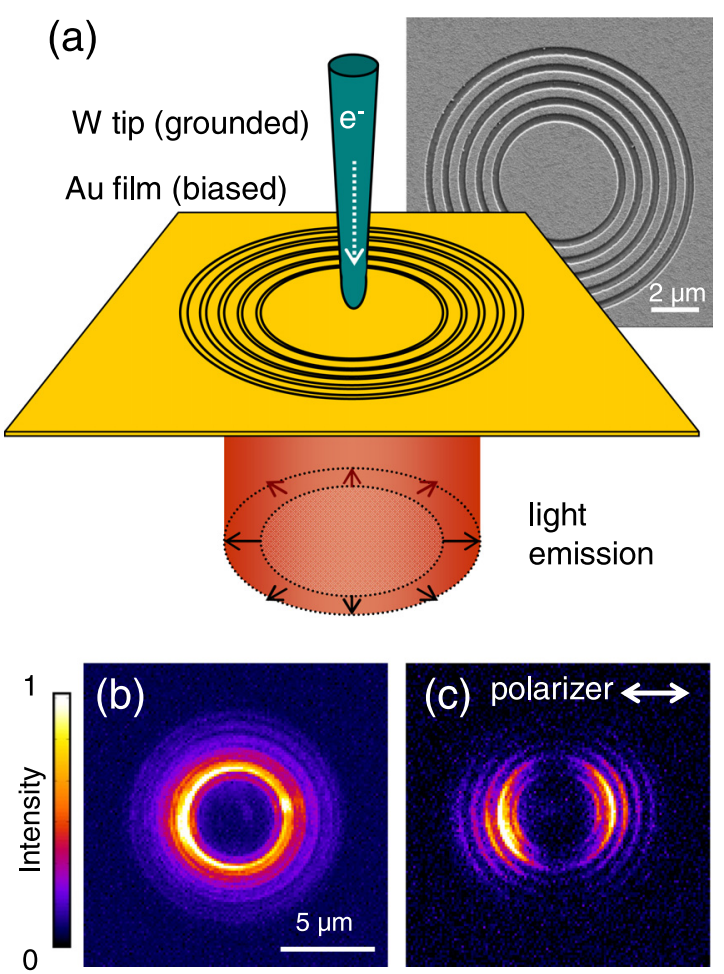

FIG. 1. (a) Schematics of the experiment: the local, electrical excitation of the SPs on a plasmonic lens using the tip of an STM leads to the emission of a cylindrical light beam with radial polarization (inset: scanning electron micrograph of the plasmonic lens). (b) Real-plane optical image in false color of the emitted light collected through the substrate with a high-NA (1.45) objective lens. (c) same as (b) but filtered with a linear polarizer (the white double arrow indicates the transmission axis). 
Our experimental setup consists of an STM head mounted on an inverted optical microscope. ${ }^{14,15} \mathrm{~A}$ nanopositioning stage controls the sample position between the tip of the STM and a high numerical aperture oil-immersion objective lens $(\mathrm{NA}=1.45)$. The plasmonic structure is prepared by focused ion beam (FIB) etching of a gold film, $200 \mathrm{~nm}$ in thickness, thermally evaporated on a glass coverslip coated with a $100 \mathrm{~nm}$-thick layer of indium tin oxide (ITO). The design of the structure is either a 5-slit circular grating or a single circular slit, with the following parameters: slit width $300 \mathrm{~nm}$, etch depth $200 \mathrm{~nm}$, and grating period $700 \mathrm{~nm}$. Structures with central disks of inner diameters $D_{i n}=1,2,3,4,5$, and $6 \mu \mathrm{m}$ are examined. STM tips are electrochemically etched tungsten wires. The sample is biased to $2.8 \mathrm{~V}$ while the tip is grounded (setpoint tunnel current in the range of 1-6nA). Inelastic tunneling of the electrons from the tip to the sample leads to the excitation of two-dimensional circular SP waves that propagate isotropically away from the tip location. ${ }^{14}$ The gold film is thick enough so as to prevent SP leakage radiation in the glass; thus, only the light from SPs scattered at the slits of the structure is detected below the substrate. The collected light is imaged on a cooled CCD camera, either in the real plane or in the Fourier plane, thus providing the spatial and the angular emission distributions, respectively.

Figures 1 and 2 show experimental results obtained with a 5-ring circular grating plasmonic lens with a inner diameter $D_{i n}=5 \mu \mathrm{m}$ (i.e., outer diameter is $11.2 \mu \mathrm{m}$ ). The real-plane image in Fig. 1(b) shows the spatial distribution of the emitted light obtained when the STM tip is located in the center of the plasmonic lens. The light emission is distributed with cylindrical symmetry over the five slits; the emission intensity rapidly decreases in an exponential manner in the outer slits. This is expected since SP scattering at the slits greatly reduces the SP propagation length. We then measure the real-plane image under similar conditions but with a linear polarizer in front of the CCD camera (the polarization axis is oriented as shown in Fig. 1(c)). Total extinction along the orthogonal axis indicates that the polarization of the collected light is purely radial.

When the tip is centered on the plasmonic lens, a small and intense doughnut-shaped spot is seen in the center of the Fourier plane (see Fig. 2(b)). Figure 2(f) shows the same experiment, but with a linear polarizer placed in front of the CCD camera. These results clearly demonstrate the emission of a highly collimated beam of purely radial polarization propagating perpendicularly to the sample plane. From the polar plot below Fig. 2(b), the angular divergence of the beam is measured at $\pm 3.8^{\circ}$ (half width at half maximum (HWHM) of the intensity profile). This is at least three times lower than previously reported values for other electrically driven microscale sources of $\mathrm{CV}$ beams (e.g., $\pm 11.5^{\circ}$ for a VCSEL source ${ }^{11}$ or $\pm 37^{\circ}$ for a metallic cavity nanolaser ${ }^{10}$ ). Remarkably, the angular divergence of our microscale light source is also much lower than that which has been reported so far for vertically emitting plasmonic antennas such as patch,${ }^{16}$ aperture, ${ }^{17}$ and bull's eye optical antennas ${ }^{18}$ which are driven by emitter or waveguide near-field coupling. This effect is both due to the design of our plasmonic lens and to the extreme spatial selectivity of the electrical excitation with tunnel electrons. ${ }^{19}$

The Fourier-plane images in Figs. 2(a)-2(d) show the angular distribution of the light emitted below the substrate. The lateral position of the STM tip on the central disk of the plasmonic lens is varied in parts (a)-(d). We also obtain
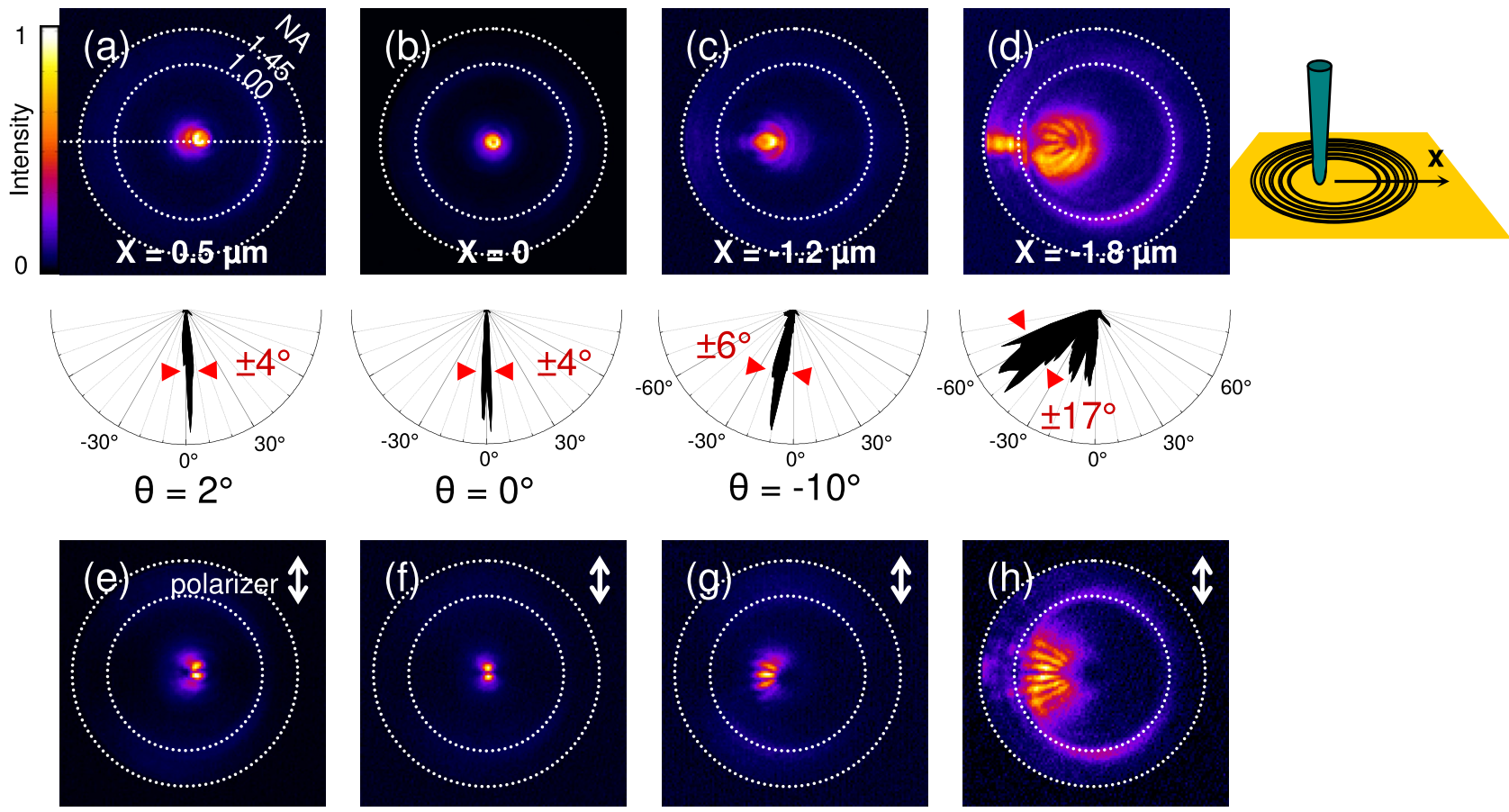

FIG. 2. Angular distribution of the emitted light. (a)-(d) Fourier-plane optical images in false color of the light detected from the same 5-slit circular grating plasmonic lens as in Fig. 1. These results are obtained under the same conditions, but for four different lateral positions of the STM excitation on the central disk region. $\mathrm{X}$ is the distance from the center of the plasmonic lens. Dotted white circles indicate the maximum acceptance angle $\left(\mathrm{NA}=1.45, \approx 73^{\circ}\right)$ and the critical angle $\left(\mathrm{NA}=1.00, \approx 41^{\circ}\right.$ ). The polar plot below each Fourier-plane image is obtained by measuring the intensity profile along the white dotted horizontal line. In the polar plots are indicated the polar angle of emission $\theta$ and the angular divergence of the beam, defined as the HWHM, rounded to the unit. (e)-(h) same as (a)-(d) filtered with a linear polarizer (the white double arrow indicates the transmission axis). 

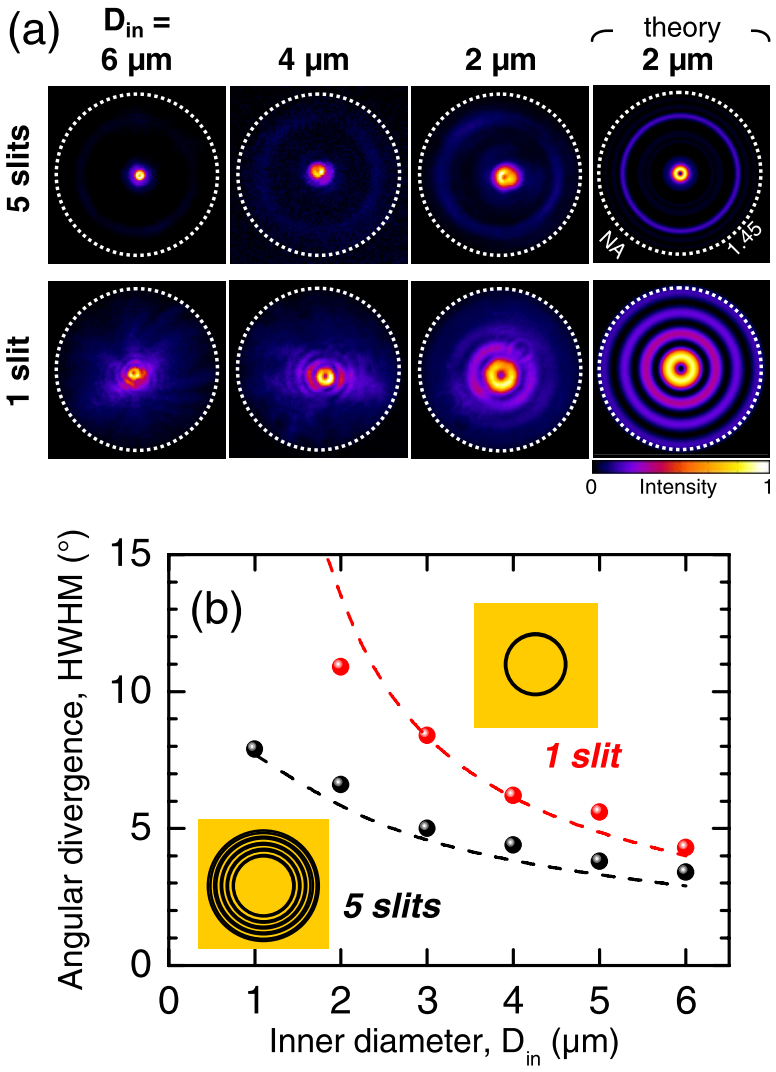

FIG. 3. Effect of the inner diameter and the number of slits in the plasmonic lens. (a) Experimental and theoretical Fourier-plane images in false color of the light from a 5-slit circular grating (top row) and a single circular slit (second row) of inner diameters $D_{i n}=6 \mu \mathrm{m}, 4 \mu \mathrm{m}$, and $2 \mu \mathrm{m}$, when the STM excitation is centered on the plasmonic lens. The dotted white circle indicates the maximum acceptance angle $\left(\mathrm{NA}=1.45, \approx 73^{\circ}\right)$. (b) Experimental and theoretical angular divergence of the emission from a 5-slit circular grating and a single circular slit, versus the inner diameter of the central disk $D_{\text {in }}$ (STM excitation in the center; solid spheres: experimental data; dashed lines: model).

Fourier-plane images under similar conditions but with a linear polarizer in front of the CCD camera, as shown in Figs. 2(e)-2(h). When the tip is $0.5 \mu \mathrm{m}$ from the center, the emitted light beam is tilted by $2^{\circ}$ from the normal and its collimation and radial polarization are preserved (see Figs. 2(a) and 2(e)). With the tip at $1.2 \mu \mathrm{m}$ from the center (Fig. 2(c)), a tilt of $10^{\circ}$ is obtained, with basically the same azimuthal divergence $\left( \pm 4^{\circ}\right)$ but a slightly higher radial divergence $\left( \pm 6^{\circ}\right)$. When beam collimation is the desired result, this technique provides a method to electrically generate a narrow beam from a microscale light source, and the ability to control the emission direction within a $0^{\circ}-10^{\circ}$ polar range and-thanks to cylindrical symmetry-a $0^{\circ}-360^{\circ}$ azimuthal range. Figure $2(\mathrm{~g})$ reveals, however, that the polarization state is no longer purely radial when the tip is moved substantially from the center. This is even more obvious with the tip at $1.8 \mu \mathrm{m}$ (i.e., only $0.7 \mu \mathrm{m}$ from the inner edge of the first slit). In Fig. 2(d), the emission is highly divergent, with a strong contribution above the critical angle. As well, the polarization-filtered image of Fig. 2(h) reveals a quite intricate polarization pattern, with several azimuthally polarized lobes below the critical angle, and an essentially radially polarized supercritical contribution.
Figure 3 shows the importance of having a plasmonic lens that consists of a circular grating rather than just a single circular slit. The effect of the inner diameter is also investigated. Figure 3(a) shows the Fourier-plane images measured for plasmonic lenses consisting of either a 5-slit circular grating (top row) or a single circular slit (second row) of inner diameter $D_{\text {in }}=6 \mu \mathrm{m}$ (first column), $4 \mu \mathrm{m}$ (second column), or $2 \mu \mathrm{m}$ (third column). When compared to each other, these images reveal that the number of slits has an important effect on the beam collimation. The emission from a single circular slit yields a second order Bessel function intensity profile in the Fourier plane, with non-negligible secondary maxima, and an intense doughnut-shaped central pattern. The existence of the secondary lobes in the emission pattern means that the collimation is degraded when only one circular slit is present. Conversely, the intensity profile in the Fourier plane for a multiple slit grating is a coherent superposition of Bessel functions with secondary maxima at different radii; therefore, only the central doughnut-shaped patterns interfere constructively and the secondary maxima cancel each other out.

We use a simple analytical model ${ }^{20}$ to simulate the Fourier-plane images of Fig. 3 and determine the theoretical angular divergence of the emitted light. The SP scattering into photons at the slits is modeled as the radiation of multiple electric dipoles located along circles of the same diameter as the circular slits. We then calculate the complex electric field vector of these dipoles in the far field. Due to the subwavelength width of the slit, we assume that only the in-plane radial field component is scattered toward the substrate; hence the dipoles in the model are horizontally oriented along the radial direction. When the tip is centered, all the dipoles along each circle emit in phase and with the same amplitude. In the model, we set a relative weight on the contribution of the five circles that exponentially decreases with their diameter. Figure 3(a) (fourth column) shows the resulting Fourier-plane images calculated for the 5-slit circular grating and for the single circular slit, with an inner diameter $D_{i n}=2 \mu \mathrm{m}$. These calculated results reproduce well the experimental Fourier-plane images (third column, Fig. 3(a)). Figure 3(b) shows good agreement between the experimental and theoretical angular divergence of the light emitted from a 5-slit circular grating and a single circular slit. We note, however, that the model overestimates the angular divergence for single circular slits with the smaller central disk diameters, probably because the width of the slit (not taken into account in the model) becomes non-negligible as compared to the diameter of the structure. In summary, a plasmonic lens consisting of a single circular slit may be preferable when the smallest total size of the structure is desired; however, a structure with multiple concentric slits yields better beam collimation with lower divergence and improved damping of secondary lobes. Moreover, it is expected that the efficiency of the SP scattering into light is significantly higher for a 5-slit circular grating than for a single circular slit, a crucial point for the optimization of the emission flux of a light source.

In conclusion, we demonstrate an electrically driven, microscale light source emitting radially polarized beams with very low angular divergence $\left( \pm 4^{\circ}\right)$. The basis of this 
light source is a plasmonic lens, whose SPs are locally excited through the inelastic tunneling of low-energy electrons from the tip of an STM. This excitation method may be described by the radiation of a vertical electric dipole inside the tip-sample gap. ${ }^{19}$ This makes it ideal for plasmonic structures with cylindrical symmetry. Furthermore, this excitation method may be integrated with current microelectronic technology, since it requires only low voltage $(<3 \mathrm{~V})$, low current (nA), and may be adapted on a chip as a planar tunnel junction. (This is an important advantage over other local SP excitation methods which use high-energy electron beams or near-field optical probes). We expect that our technique may be extended to the generation of many other kinds of CV beams, simply by changing the design of the pattern etched in the gold film. In particular, 2D chiral structures such as spirals may be used to generate light beams that carry optical orbital angular momentum. Such light beams have promising applications in the manipulation of nano-objects. ${ }^{21}$ It should also be noted that the emission of a radially polarized beam through the local SP excitation in the center of a plasmonic lens constitutes the perfect time-reversed process of the SP focusing of a radially polarized beam from the farfield. 13,22,23 Thus, in order to produce a "designer light beam," the suitable pattern to be etched in the gold film may be found for virtually any desired emission pattern through a numerical time-reversal procedure. ${ }^{24}$

This work was supported by the Région Ile-de-France (C'Nano IdF, DIM Nano-K, "COSSMET" project) and Université Paris-Sud (Grant “Attractivité 2013”). The financial support of China Scholarship Council (CSC) (No. 201304910386) is gratefully acknowledged. The authors thank Thierry Fournier and Jean-François Motte at the NanoFab facility in Grenoble for the fabrication of the plasmonic structures.
${ }^{1}$ Q. Zhan, Adv. Opt. Photonics 1, 1 (2009).

${ }^{2}$ O. G. Rodríguez-Herrera, D. Lara, K. Y. Bliokh, E. A. Ostrovskaya, and C. Dainty, Phys. Rev. Lett. 104, 253601 (2010).

${ }^{3}$ M. Neugebauer, T. Bauer, P. Banzer, and G. Leuchs, Nano Lett. 14, 2546 (2014).

${ }^{4}$ C. Gabriel, A. Aiello, W. Zhong, T. G. Euser, N. Y. Joly, P. Banzer, M. Förtsch, D. Elser, U. L. Andersen, C. Marquardt, P. S. J. Russell, and G. Leuchs, Phys. Rev. Lett. 106, 060502 (2011).

${ }^{5}$ C. J. R. Sheppard and A. Choudhury, Appl. Opt. 43, 4322 (2004).

${ }^{6}$ Q. Zhan, Opt. Express 12, 3377 (2004).

${ }^{7}$ C. Maurer, A. Jesacher, S. Fürhapter, S. Bernet, and M. Ritsch-Marte, New J. Phys. 9, 78 (2007).

${ }^{8}$ E. Lombard, A. Drezet, C. Genet, and T. W. Ebbesen, New J. Phys. 12, 023027 (2010).

${ }^{9}$ H. J. Lezec, Science 297, 820 (2002).

${ }^{10}$ K. Ding, L. Yin, M. T. Hill, Z. Liu, P. J. van Veldhoven, and C. Z. Ning, Appl. Phys. Lett. 102, 041110 (2013).

${ }^{11}$ B.-Z. Xu, J.-T. Liu, L.-K. Cai, H.-F. Hu, Q. Wang, X. Wei, and G.-F. Song, Chin. Phys. Lett. 30, 034206 (2013).

${ }^{12}$ L. Cai, J. Zhang, W. Bai, Q. Wang, X. Wei, and G. Song, Appl. Phys. Lett. 97, 201101 (2010).

${ }^{13}$ W. Chen, D. C. Abeysinghe, R. L. Nelson, and Q. Zhan, Nano Lett. 9, 4320 (2009).

${ }^{14}$ T. Wang, E. Boer-Duchemin, Y. Zhang, G. Comtet, and G. Dujardin, Nanotechnology 22, 175201 (2011).

${ }^{15}$ E. Le Moal, S. Marguet, B. Rogez, S. Mukherjee, P. Dos Santos, E. BoerDuchemin, G. Comtet, and G. Dujardin, Nano Lett. 13, 4198 (2013).

${ }^{16}$ C. Belacel, B. Habert, F. Bigourdan, F. Marquier, J.-P. Hugonin, S. Michaelis de Vasconcellos, X. Lafosse, L. Coolen, C. Schwob, C. Javaux, B. Dubertret, J.-J. Greffet, P. Senellart, and A. Maitre, Nano Lett. 13, 1516 (2013).

${ }^{17}$ A. Yaacobi, E. Timurdogan, and M. R. Watts, Opt. Lett. 37, 1454-1456 (2012).

${ }^{18}$ H. Aouani, O. Mahboub, N. Bonod, E. Devaux, E. Popov, H. Rigneault, T. W. Ebbesen, and J. Wenger, Nano Lett. 11, 637 (2011).

${ }^{19}$ P. Johansson, Phys. Rev. B 58, 10823 (1998).

${ }^{20}$ O. Mollet, G. Bachelier, C. Genet, S. Huant, and A. Drezet, J. Appl. Phys. 115, 093105 (2014).

${ }^{21}$ Y. Gorodetski, A. Drezet, C. Genet, and T. W. Ebbesen, Phys. Rev. Lett. 110, 203906 (2013).

${ }^{22}$ Z. Liu, J. M. Steele, W. Srituravanich, Y. Pikus, C. Sun, and X. Zhang, Nano Lett. 5, 1726 (2005).

${ }^{23}$ B. Lee, S. Kim, H. Kim, and Y. Lim, Prog. Quantum Electron. 34, 47 (2010).

${ }^{24}$ G. Leuchs and M. Sondermann, Phys. Scr. 85, 058101 (2012). 